\title{
Pulse deinterleaving based on fusing PDWs and PRI extraction process for radar-assisted edge devices considering computational costs
}

\author{
Hamid Hasani ${ }^{1}$ and Mohammad R. Khosravi ${ }^{2^{*}}$
}

\author{
${ }^{*}$ Correspondence: \\ m.khosravi@mehr.pgu.ac.ir \\ 2 Department of Computer \\ Engineering, Persian Gulf \\ University, Bushehr, Iran \\ Full list of author information \\ is available at the end of the \\ article
}

\begin{abstract}
Deinterleaving or radar pulse separation is a very important goal in terms of radar sources for identifying and implementing electronic warfare systems. In order to separate radar pulses, parameters measured by electronic warfare receivers such as electronic warfare support measures (ESM) and electronic signals intelligence (ELINT) are used in pulse separation. This paper presents a multi-parameter improved method for separating the pulse sequence of radar signals based on time of arrival (TOA) processing with sorting the other pulse descriptor words (PDW) parameters. In the proposed method, after extracting all the pulse repetition intervals (PRIs) based on TOA, the parameters of the angle of arrival, pulse width and carrier frequency (RF) are being used in pulse sorting to separate the received interleaved pulse sequences. The sequential difference histogram (SDIF) algorithm or cumulative difference histogram (CDIF) algorithm is used to extract all pulse repetition intervals. Also, in order to separate the sequence of the received pulses from all surroundings emitters, in addition to matching the potential PRI among the TOAs of the pulses and the similarity measurement in the other parameters of the pulse sequence (pulse sorting) have been used. This proposed algorithm is implemented in the integrated and complete design for deinterleaving of the radar pulses. The proposed method by considering low-cost computing sources considers a fast and low-complexity solution that can be used for edge-enabled distributed processors in aerial radar platforms as edge devices for military/combat unmanned aerial vehicles or networked missiles. The simulation results show that our method is completely effective.
\end{abstract}

Keywords: Electronic warfare receiver, Clustering, Pulse deinterleaving, Pulse descriptor word (PDW), Histograms, Pulse repetition interval (PRI)

\section{Introduction}

Nowadays, radar pulse separation or deinterleaving is a very important goal for identifying radar sources and implementing electronic warfare systems in edge devices, for example the ground-based devices which should provide electronic warfare support measures or aerial vehicles which are in connection to autonomously networked UAVs and missiles. The general concept of interleaving and deinterleaving of radar pulse trains is shown in Fig. 1. In order to separate radar pulses, parameters measured by electronic

C) The Author(s), 2021. Open Access This article is licensed under a Creative Commons Attribution 40 International License, which permits use, sharing, adaptation, distribution and reproduction in any medium or format, as long as you give appropriate credit to the original author(s) and the source, provide a link to the Creative Commons licence, and indicate if changes were made. The images or other third party material in this article are included in the article's Creative Commons licence, unless indicated otherwise in a credit line to the material. If material is not included in the article's Creative Commons licence and your intended use is not permitted by statutory regulation or exceeds the permitted use, you will need to obtain permission directly from the copyright holder. To view a copy of this licence, visit http:// creativecommons.org/licenses/by/4.0/. 


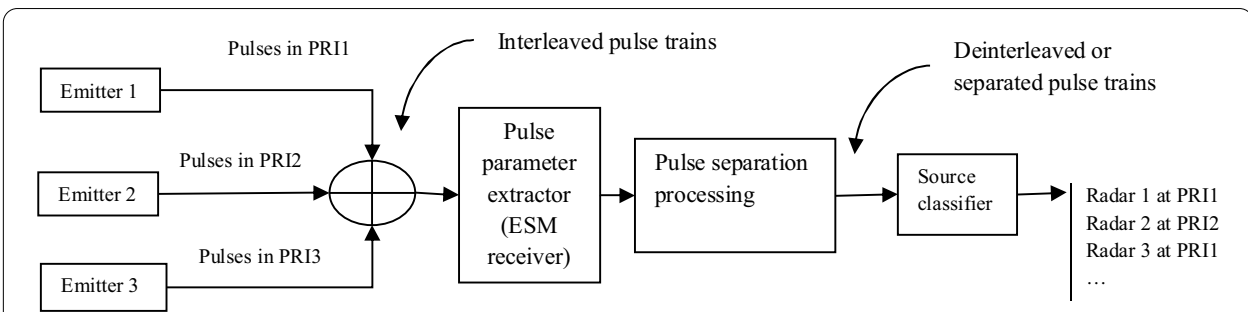

Fig. 1 Definition of the pulse interleaving and deinterleaving

warfare receivers such as electronic support measures (ESM) and electronic signals intelligence (ELINT) are used $[1,2]$. These receivers measure the parameters that describe the pulse specification (PDWs). For separation of the radar pulses, the measured parameters by electronic warfare receivers must be processed. These measured parameters are called pulse descriptor word (PDW), which have the specific properties of the emitter of the pulse source and include the parameters of time of arrival (TOA), angle of arrival (AOA), carrier frequency (RF), pulse width (PW) and pulse amplitude (PA) [1].

So far, various approaches for deinterleaving are discussed. The first methods of pulse separation were included "automatic processing and decision-making techniques" [1] and "automatic sorting of radar signals (deinterleaving)" [3]. Then newer algorithms based on PRI estimation (TOA-processing) were presented that called "cumulative difference histogram (CDIF)" and "The sequential difference histogram (SDIF)" [4-10]. In the following, the newer radar signal sorting methods using neural network algorithms and fuzzy art classification were also proposed [11-15]. These various methods have been proposed to separate the pulses by using PDWs [16-22]. In general, pulse separation methods are divided into two general methods which are pulse separation based on TOA processing and categorization of pulses based on clustering algorithms (signal sorting) [11-14, 23].

Also, because of the progress of the transmitters in sending complex pulses with different modulations and increasing the number of radar emitters, pulse separation of radar emitters has become difficult. Therefore, it is possible that this complexity can lead to each one of the pulse separation methods based on TOA-processing or pulse sorting (clustering) is not being efficient lonely. The TOA parameters of any pulse sequence have the information about the emitter's PRI [2, 24], which can be in moods of fixed, staggered, jittered, etc. So, pulse separation of the emitters that use PRI modulations such as jittered and staggered PRI will be difficult with the only-TOA processing method. Also, due to the excessive increase of emitters in the environment, it is possible that several emitters exist with similar RF and PW in the surrounding environment, which signal sorting methods may not be effective lonely. Therefore, it is necessary to use the multiparameter pulse separation algorithms with the combination of the TOA-based processing algorithms and pulse sorting (classification). In this paper, the optimized pulse separation algorithm is presented. This proposed algorithm performs pulse separation operations with several parameters simultaneously with high-performance quality.

In $[1,5,25-28]$, pulse separation algorithms are performed based on TOA processing as a part of a multi-parameter algorithm after classifying the pulses in other PDW parameters. Due to important information that lies on TOA, mostly pulse sorting by 
using the parameters except for the TOA cannot be useful, and also pulse separation algorithm based on only-TOA processing does not have high separation quality. In this paper, pulse separation using all parameters will be presented simultaneously in one algorithm. The proposed algorithm is a multi-parameter pulse separation whit "the TOA processing" algorithm that also does "pulse sorting" within itself.

At first, a different general structure of multi-parameter pulse separation is presented effectively and attempt to use all the parameters usefully based on an efficient sequence search algorithm that has fewer errors in the sequence search. In the consecutive processes, this improved sequence search prevents the splitting of the pulse sequence of the emitters to the sub-sequences and also extracts the pulse sequence of the staggered-PRI emitters in one pulse sequence by using the proposed multi-parameter sequence search. And there is no need for staggered sequence detection analyzes which have been performed in previous methods $[4,29,30]$.

The comprehensive structure of the electronic warfare systems and the general scheme of the processes required for the pulse separation operations is presented in Fig. 2.

This paper is organized as follows: all the required materials for understanding the proposed method are presented in the second section. The third section introduces the proposed method. The fourth section shows the simulation results. And the last section is dedicated to conclusions and future works.

\section{Foundations of TOA-based pulse separation methods}

The pulse separation methods based on the TOA-processing algorithms are performed in two stages, PRI extraction and sequence search [5-9]. The Common algorithms in association with PRI extraction are SDIF and CDIF algorithms, which are using to extract PRIs frequently due to their higher speed and quality [31-33]. The SDIF histogram algorithm is a type of TOA difference calculating algorithm and is a modified and improved version of the cumulative difference histogram (CDIF) [30]. In the SDIF

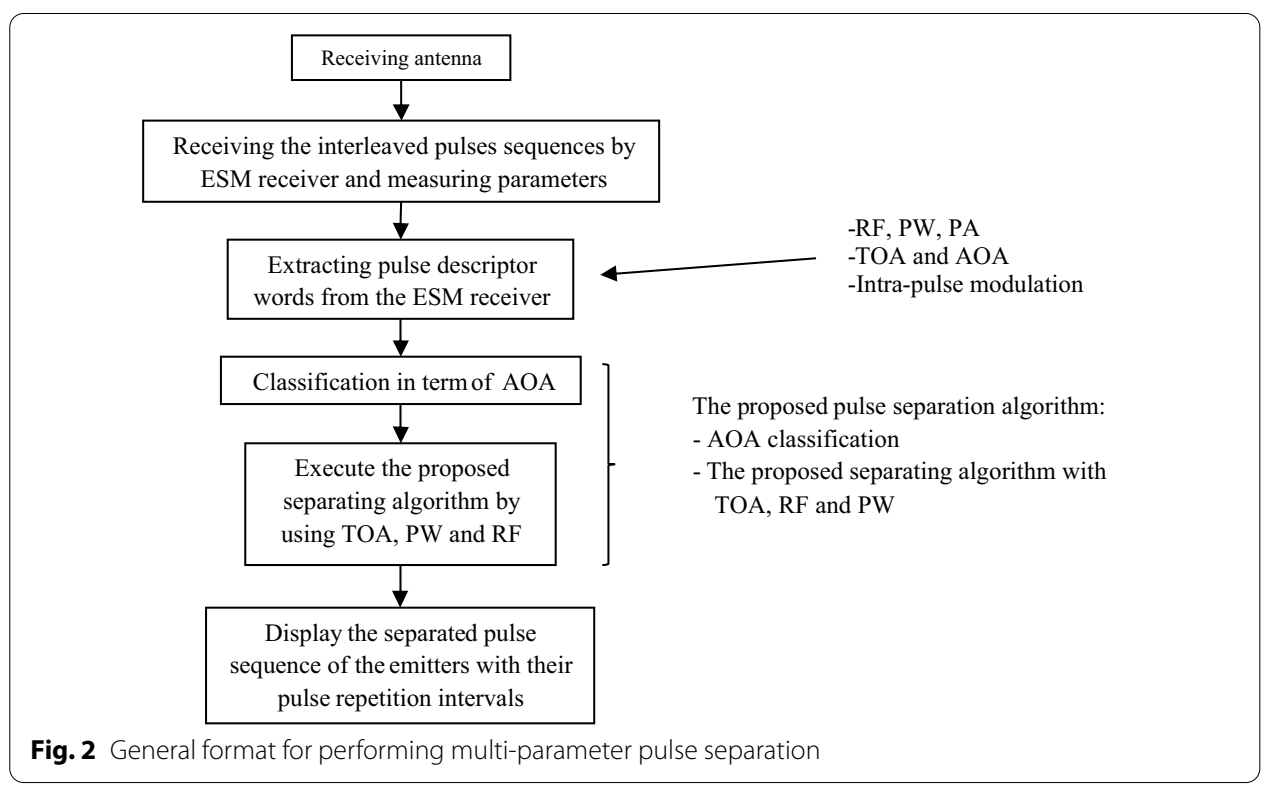


algorithm, the TOA differences are measured, among all the different received pulses at level of the difference $(C)$ and then the histogram of the TOA differences is formed to extract the potential PRIs.

$$
\operatorname{diff}_{\mathrm{TOA}}=|\mathrm{TOA}(j)-\mathrm{TOA}(j+C)|
$$

A histogram of the TOA difference values is constructed for each Bin height that each bin height corresponds to each unique calculated TOA difference. Bins with heights exceeding a particular threshold are taken as valid potential PRIs. The optimum threshold function is suggested as follows [30]:

$$
\operatorname{Thr}(\tau)=x(E-C) e^{\frac{-\tau}{k N}}
$$

In the above formula, $E$ is the aggregate number of pulses, $N$ is the number of available bins in the histogram, and $C$ is the order of difference. The optimum values for $x$ and $k$ are obtained empirically [30].

After extracting the potential PRIs, the sequence search operation is performed. In [6], a method for sequence search based on PRI and TOA matching had been suggested. (This means that the algorithm finds the appropriate potential PRI between two pulses with different TOAs.) Based on the sequence search algorithm by matching the potential PRI between the pulse TOAs, in the case of staggered PRI and jittered PRI sequences, the separation of the pulse sequences is too difficult, using the sequence search algorithm in [6]. By using SDIF or CDIF algorithms, the potential PRI for the pulse separation algorithms is extracted.

In [5], the idea of pulse separation was introduced, using PW and TOA in the sequence search. In this way, first, the classification is done in terms of the AOA, then PRI analysis is performed using two-dimensional sequence search using matching TOA and PW, and finally, the carrier frequency analysis has performed the categories. In this paper, for the pulse sequence separation of the emitters, an improved method is performed with better performance and fewer processing blocks by using a multi-parameter sequence search. By implementing the proposed algorithm, the pulse sequences are separating by matching the appropriate potential PRIs between the pulses TOAs and measuring and comparing the similarity in other parameters of pulses (PW, RF, and AOA). The concepts and general scheme of the proposed method are presented in the next section.

\section{The proposed method}

In this paper, modifications made to the general scheme of the pulse separation included PRI extraction and sequence search presented in $[5,6]$, which increases the efficiency of the pulse separation algorithm. This operation does this by describing the new variables for PRI matching among the TOAs and simultaneously examining and comparing the similarity in other parameters of each emitter pulse sequence. In this proposed algorithm, to extract the potential PRIs, the SDIF algorithm and the threshold function are used [5]. Then, by using the proposed sequence search algorithm, the pulse sequences of the emitters are separated. The input parameters of the algorithm are the PDWs of a time frame ( $T_{i}$ seconds) of the interleaved pulse sequences. These pulses are being received from all surrounding emitters located at a similar AOA and 
contain the $N$ number of pulses. The histogram of the TOAs differences is formed in the SDIF algorithm. Then the threshold set on the histogram and each histogram value that exceeds the threshold is considered as the potential PRI. And after the harmonic testing, the potential PRI is used for the sequence search operation. The level of difference starts from one and is added to higher levels if necessary $(C=1)$. After extraction of the potential PRIs, the first change in the general separation process is that the potential PRIs divided into two categories, jittered and non-jittered PRI, depending on their characteristics. After dividing these potential PRIs, the procedure of the sequence search can be performed on them separately. The separation of potential PRIs will have a significant impact on improving the performance of the sequence search. Adjacent values crossing the threshold, considered as jittered potential PRIs. So, instead of jittered PRIs average, all the values crossing the threshold are used in the sequence search.

$$
\frac{\operatorname{PRI}_{\mathrm{Jitter}}\left(\tau+t_{\mathrm{Bin}}\right)}{\operatorname{PRI}_{\mathrm{Jitter}}(\tau)}=1+\varepsilon \quad \forall 0<\tau<t_{\mathrm{Bin}}
$$

In the above formula, $t_{\mathrm{Bin}}$ is the time interval of a bin of the bar chart and $\varepsilon$ is a small number. In both modes of the jittered and non-jittered potential PRIs, harmonics or coefficients PRI may observe, resulting from the missing pulses and or levels of difference [4]. For example, when a pulse is lost, it is possible that differences in the PRI coefficients created in the histogram, which may exceed the threshold if the number of such missed pulses is a lot. These harmonics must remove if observed. If a pulse sequence PRI possibly equal harmonic PRI of another pulse sequence, after the first pulse sequence is extracted (smaller PRI), the removed potential PRI appears again in another subtraction, then its pulse sequence is extracted. Therefore, the steps of the separation algorithm are shown as follows (Fig. 3).

1. Receiving the input pulse train (number of $N$ pulses) sorted by TOA, and the TOA differences calculated from the level difference $C=1$.

2. When the number of these pulses is greater than the initial value $N_{0}$, the processing is performed on the input.

3. The SDIF histogram with the level of difference $C$ formed by applying the threshold and the histogram values that passed the threshold, selecting as the potential PRI.

4. Harmonics or PRI coefficients [4] checked, then the missed pulses effect eliminated by removing the coefficients of potential PRI of two, three, etc.

5. The potential PRIs are divided into two categories, jittered potential PRI and nonjittered potential PRI, and a sequence search is performed on each of the PRI categories.

6. If the sequence search on the potential PRIs is successful, the difference level set $C=1$, the separated pulse sequence is extracted from the input sequence as a pulse sequence of an emitter.

7. If the sequence search fails, the level of difference value increases $(C=C+1)$ one unit, then for the new level of difference value, the process is repeated from the second step. 


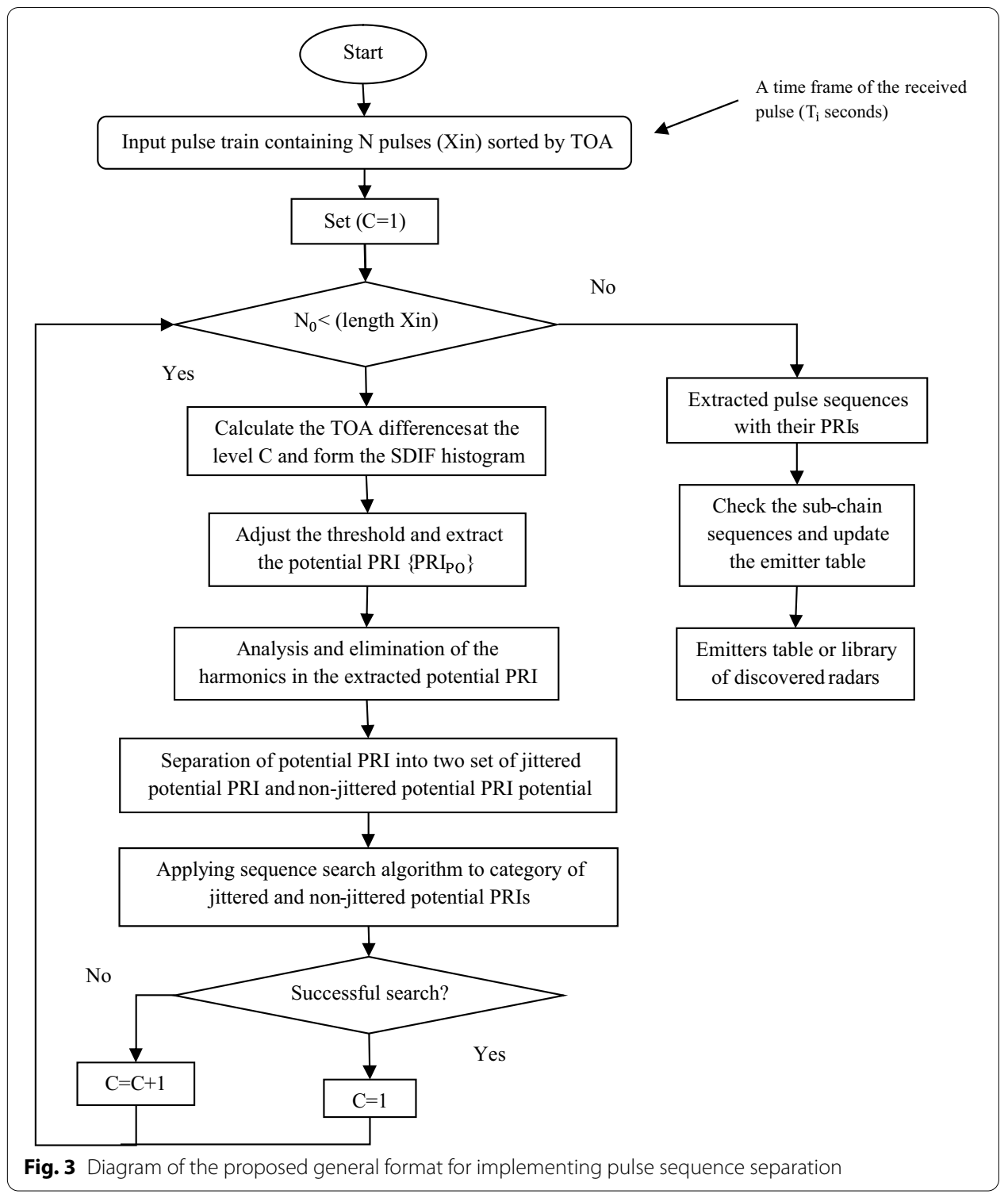

8. Steps two to seven repeat until all the sequences extracted or the number of input pulses is less than $N_{0}$ ( $N_{0}$ is a small number of pulses that remain in the original pulse sequence and are not extracted).

After extracting potential PRIs, the sequence search operation performs to separate the pulse trains of all emitters. The proposed sequence search algorithm uses the potential PRIs, carrier frequency and pulse width to separate all the pulse sequences. In this section, the two operations "classification in terms of carrier frequency and pulse width" and "matching the appropriate potential PRIs among the TOA of the pulses" are performed simultaneously. Each potential PRIs that crossed the threshold means that a large number of the pulses have such differences among theirs TOAs, but all of them do not indicate the TOAs differences among the members of the actual pulse sequence. Therefore, if the sequence search is based on only TOA-processing, there is a possibility 
that the actual pulse sequences broke into several subsequences, and the extracted pulse sequence represents a false emitter. Therefore, the sequence search based on only the TOA-processing maybe leads to an error in the sequence search. Therefore, using the TOA with the other parameters such as the angle of arrival, pulse width, or carrier frequency, the sequence search errors can be reduced, which associates with vital in mobile computing [34-37] in electronic warfare. For the Implementation of the pulse separation, the proposed algorithm performs in a two-step sequence search that executes in the form of a flowchart shown in Figs. 4 and 5. In the first stage of the sequence search, if the first three or four sentences of the sequence extracted by using potential PRIs, then the sequence search process is continued in the second stage. The success of sequence search in the first stage means the existence possibility of a sequence, and then following of the sequence search performs in the second stage according to the flowchart in Fig. 5. The difference of the second stage is considering the effect of missed pulses in the sequence search.

For the sequence search implementation procedure, the functions $Z_{k}(i, j)$ and $M_{k}(j)$ are defined. The function $Z_{k}(i, j)$ is used in the sequence search algorithm to match the potential PRIs among the pulses TOAs. Also, the function $M_{k}(j)$ used to compare the

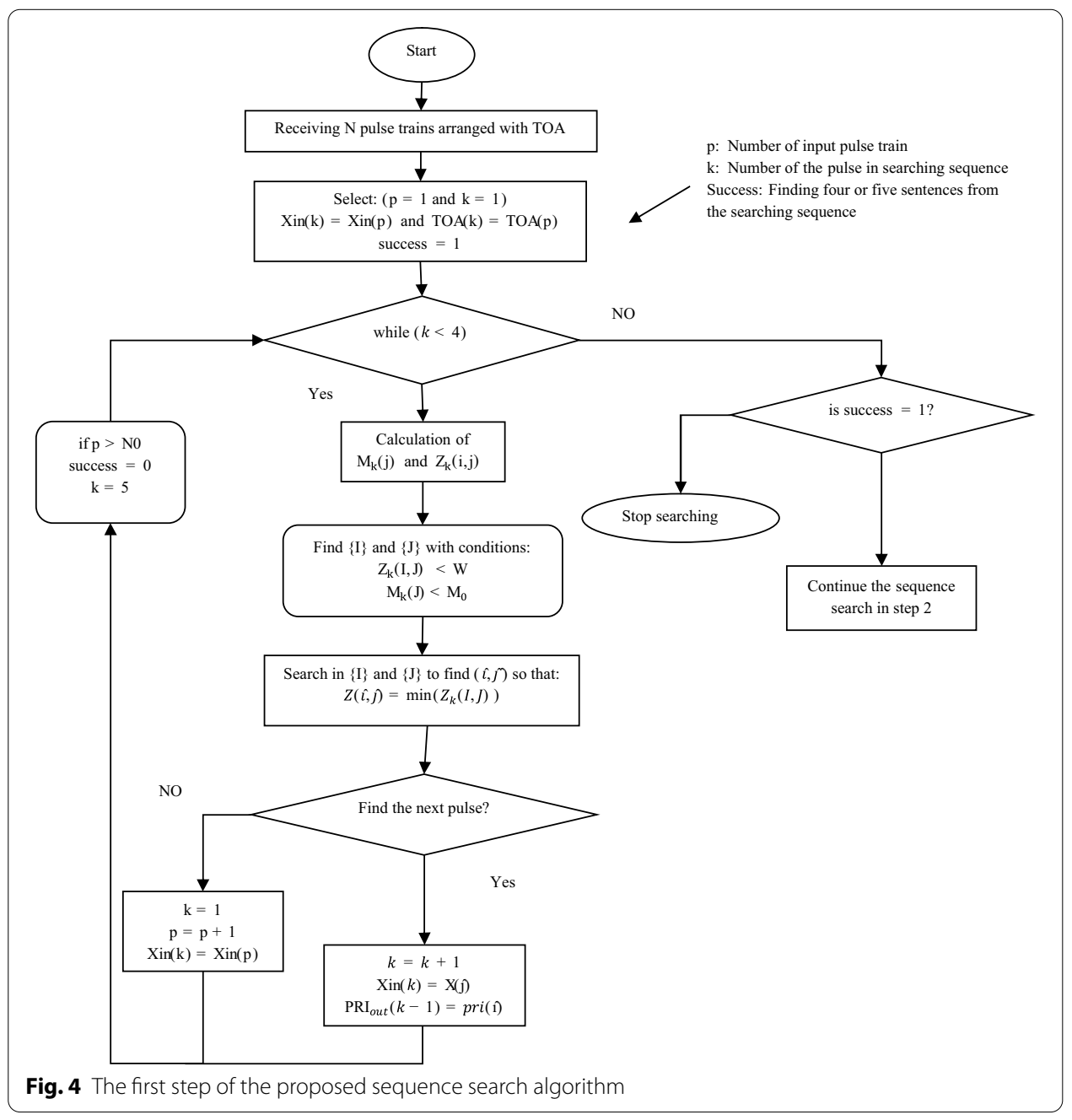




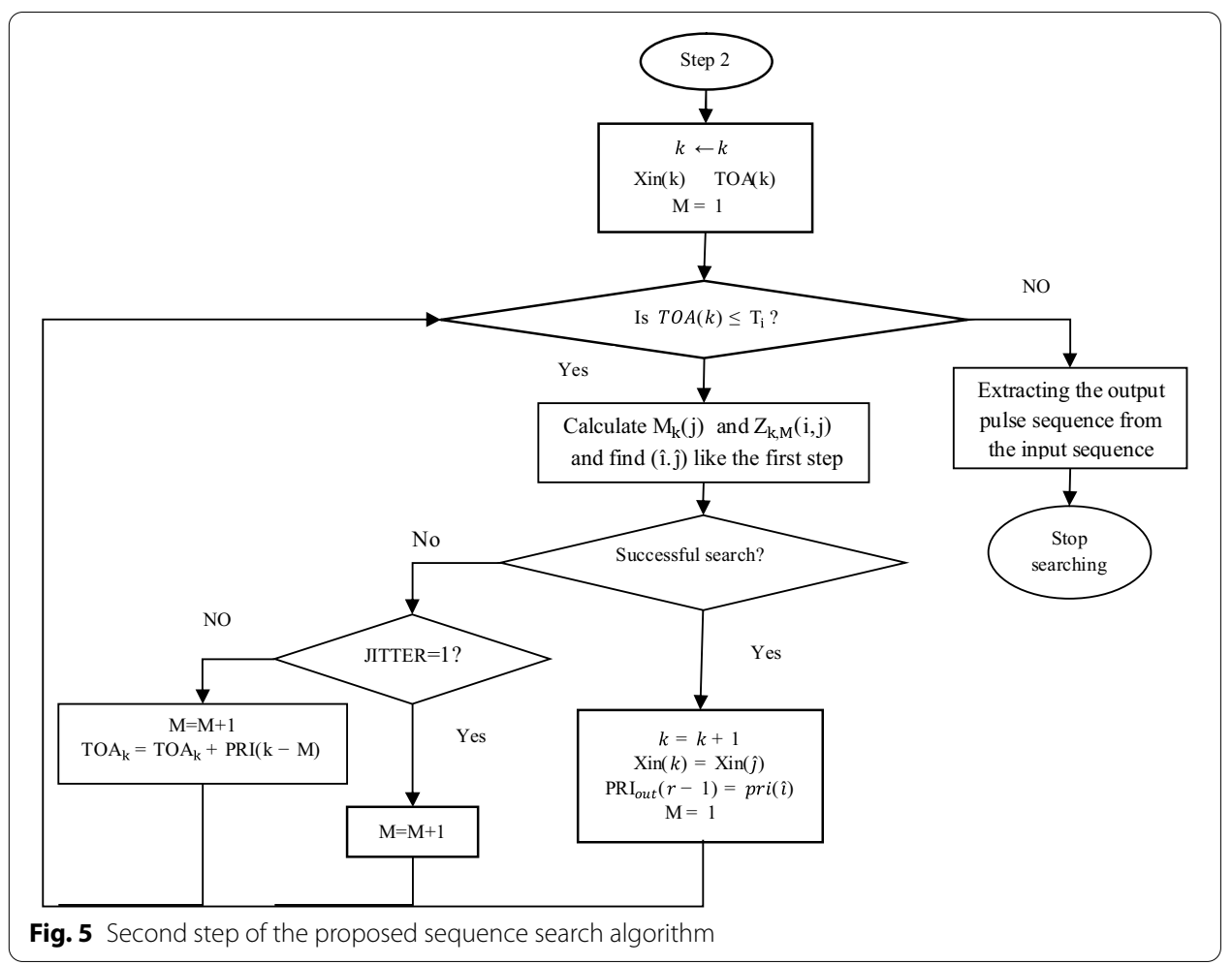

other parameter's similarity includes carrier frequency and pulse width of the extracting sequence. Among all the pulses with the most similarity to each other, the sequence search algorithm will find the next pulse of the sequence if the potential PRIs match among TOAs of the last extracted pulse and the next pulse. If $N_{\mathrm{P}}$ is the number of the potential PRIs (PRI $)$ exceeding the threshold, so:

$$
\operatorname{PRI}_{\mathrm{p}}=\left[\operatorname{PRI}_{\mathrm{p}}(1), \operatorname{PRI}_{\mathrm{p}}(2), \operatorname{PRI}_{\mathrm{p}}(3), \ldots, \operatorname{PRI}_{\mathrm{p}}\left(N_{\mathrm{p}}\right)\right]^{T}
$$

Now, if $(j=k)$ is the last pulse extracted from the received pulse sequence, therefore, the sequence search will find the next pulse $(j=k+1)$ of the sequence by TOA and PRI matching as follows.

$$
\begin{aligned}
& \mathrm{TOA}_{\text {diff }}(j, k)=\left[\left|\mathrm{TOA}_{j}-\mathrm{TOA}_{k}\right|\right]_{N_{\mathrm{p}} \times 1} \\
& Z_{k}(i, j)=\mathrm{PRI}_{\mathrm{p}}(i)-\mathrm{TOA}_{\text {diff }}(j, k) \quad 1 \leq i \leq N_{\mathrm{p}} \quad k+1 \leq j \leq N
\end{aligned}
$$

The function $M_{k}(j)$ is used to investigate the parametric similarity of the pulses of the sequence. This function is taken from the metric spatial distance. The parameters of carrier frequency and pulse width are used in this function [38]. In this function, $\mathrm{RF}_{0}$ and $\mathrm{PW}_{0}$ are used to adjust the maximum acceptable radius of parameters in 
pulse-to-pulse variations in a sequence. Thus, the criterion function of matching the parameter similarity is obtained by using the following formula:

$$
M_{k}(j)=\frac{\left(\mathrm{RF}_{j}-\mathrm{RF}_{k}\right)^{2}}{\mathrm{RF}_{0}^{2}}+\frac{\left(\mathrm{PW}_{j}-\mathrm{PW}_{k}\right)^{2}}{\mathrm{PW}_{0}^{2}}
$$

Other functions such as Euclidean distance and internal multiplication can be used to measure the similarity of the pulse parameters with each other.

After performing calculations of $Z_{k}(i, j)$ and $M_{k}(j)$, the collections $\{I\}$ and \{\} are obtained as follows. These collections are applied to find the next pulse of the extracting sequence and matching the appropriate PRI.

$$
\begin{aligned}
& Z_{k}(J, I)<W \\
& M_{k}(J)<M_{0}
\end{aligned}
$$

In the above formula, $W$ is a maximum interval that the potential PRIs match among the TOAs of the last extracted pulse and the next pulse in the searching sequence, which is selected practically. And $M_{0}$ adjust the maximum acceptable distance of the parameters of two pulses of an emitter in terms of pulse-by-pulse similarity.

Then, by searching in two collections $\{I\}$ and \{\} the next sentence of the searching sequence $(\hat{J})$ and the potential PRI that matching the time of arrival between the last extracted pulse and the next pulse of the searching sequence $(\hat{I})$ are determined as follows.

$$
(\widehat{J}, \widehat{I})=\min _{I, J}\left(Z_{k}(I, J)\right)
$$

If the next sentences are found, the sequence search will continue and the output PRI is calculated as follows:

$$
\mathrm{PRI}_{\mathrm{out}}=\operatorname{TOA}(\widehat{J})-\mathrm{TOA}(k)
$$

Sometimes in the second step of the sequence search, the next pulse of the sequence is not found, and then this pulse is considered a missed pulse. Therefore, the sequence search will continue to find the next pulse after the missed pulse. So, the difference between the second and first stage of the sequence search is considering the effect of missing or undiscovered pulses by the ESM receiver. So, to prevent the breaking of the sequence search due to the missing pulses, changes are made to the sequence search operation. And in the mode of jittered sequence search, the PRI-TOA matching function is changed as follows.

$$
Z_{k, M}(i, j)=M\left(\mathrm{PRI}_{\mathrm{p}}(i)-\mathrm{TOA}_{\text {diff }}(j, k) \quad 1 \leq i \leq N_{\mathrm{p}}, k+1 \leq j \leq N\right.
$$

And in the mode non-jittered sequence search, the PRI matching function does not change (always $M=1$ ), but the TOA of the last $(k)$ extracted pulse of the sequence changes as follows. 
$\operatorname{TOA}_{\text {diff }}(j, k)=\operatorname{TOA}_{\text {diff }}(j, k)-\operatorname{PRI}_{\text {out }}(k-M)$

Similar to the previous case, if the algorithm finds the next pulse successfully, the $M$ value will remain fixed $(M=1)$. And if the algorithm does not find the next pulse in the sequence search, the $M$ value is added one unit to find the next pulse of the sequence after the missed pulse, and the sequence search will continue. Therefore, the second step performs as the flowchart in Fig. 5.

\section{Simulations and results}

The proposed algorithm is implemented in different PRI modes and emitter numbers and also compared with the only TOA-based processing algorithm in sequence search. It assumed that all the emitters are located at the same angle of arrival and belong to an angular category. So, the sequence search algorithm does not use the angle of arrival because of the similarity. The carrier frequency of the emitters is assumed to be agile with $10 \%$ variation around the central frequency with $5 \%$ measurement error is considered for the pulse width. The range of variation of the emitter's carrier frequency is between 0.5 and $18 \mathrm{GHz}$, and pulse width can take a value between 1 and $10 \mu \mathrm{s}$.

In the first step of the simulation, the general operation of the pulse separation algorithm is performed. At this stage of the simulation, the proposed pulse separation algorithm is implemented on the received pulses from several emitters. And therefore, to analyze the implementation procedure, the proposed algorithm is evaluated in the pulse separation of the five emitters located in the equal angular cell. So, the pulse separation algorithm uses the carrier frequency and the pulse width simultaneously with the pulse TOA, then these parameters of the received pulse are presented to the algorithm. These five emitters consist of two radars whit stationary PRI, two radars whit staggered PRI, and one jittered PRI radar. The PRI values and their types for each emitter are shown in Table 1. The ESM receiver processes the interleaved pulse sequences and measures the PDWs sorted by the TOAs, and the algorithm separates the interleaved pulse.

The pulse separation algorithm extracts the pulse sequence of all emitters, and then the PRI variation diagram of each separated pulse sequence of the emitters is obtained as follows in Fig. 6.

As shown in Fig. 6, the algorithm has separated all the sequences correctly, and the PRI of the pulse sequences extracted correctly. Also, as can be seen, the proposed sequence search algorithm separates the staggered sequences into one sequence instead of three sequences.

Table 1 Values and type of PRI of the pulses sequence received from emitters

\begin{tabular}{|c|c|c|c|c|}
\hline Emitter & PRI type & Average PRI (s & & \\
\hline A & Fixed PRI & $157 e-05$ & & \\
\hline B & Fixed PRI & $137 e-05$ & & \\
\hline C & Staggered PRI with 3-level & $106 e-05$ & $112 e-05$ & $9.08 e-04$ \\
\hline D & Staggered PRI with 3-level & $181.6 e-05$ & $205.4 e-05$ & $153.8 \mathrm{e}-05$ \\
\hline E & Jittered PRI with $10 \%$ variation & $10 e-04$ & & \\
\hline
\end{tabular}



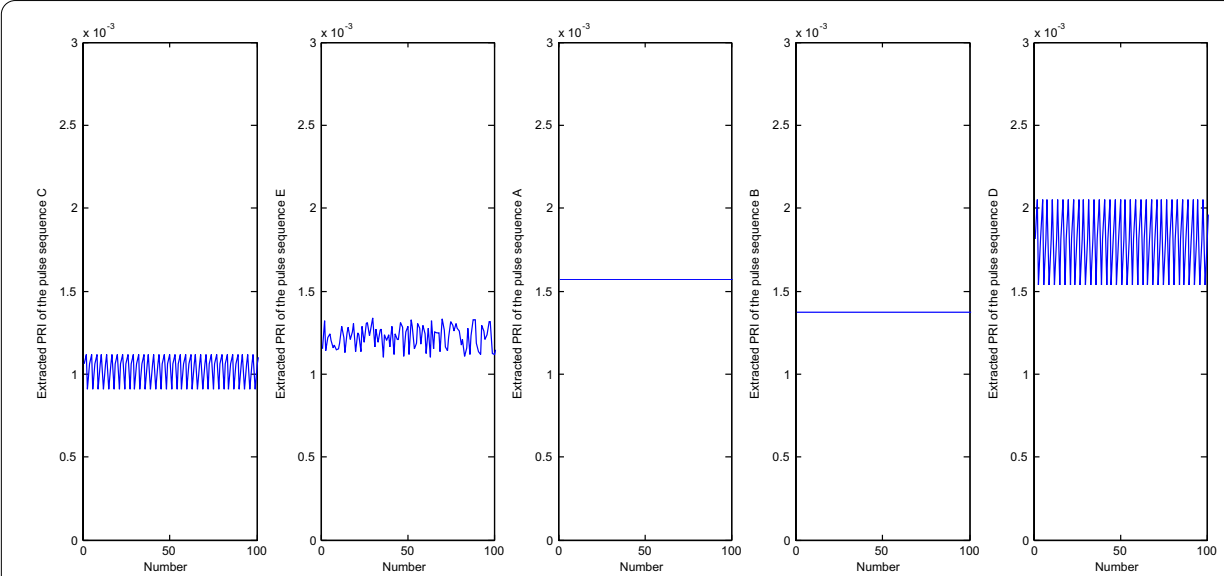

Fig. 6 Diagram of PRI changes of separated pulses sequence of emitters in the Table 1

Table 2 The result of different states investigated in the separation of interleaved pulse trains by methods $A$ and $B$

\begin{tabular}{|c|c|c|c|c|c|c|c|c|}
\hline \multirow{3}{*}{$\begin{array}{l}\text { Stage } \\
\end{array}$} & \multirow{3}{*}{$\begin{array}{l}\text { Pulse separation } \\
\text { algorithm } \\
\text { By method A }\end{array}$} & \multirow{2}{*}{\multicolumn{3}{|c|}{$\frac{\text { Correct emitters detected }}{\text { Classic/staggered/jittered }}$}} & \multirow{2}{*}{\multicolumn{3}{|c|}{$\frac{\text { False emitters detected }}{\text { Classic/staggered/jittered }}$}} & \multirow{3}{*}{$\begin{array}{l}\begin{array}{l}\text { Processing } \\
\text { time (s) }\end{array} \\
.1617\end{array}$} \\
\hline & & & & & & & & \\
\hline & & 2 & 0 & 0 & 0 & 0 & 0 & \\
\hline & By method B & 0 & 0 & 0 & 0 & 0 & 0 & .2532 \\
\hline \multirow[t]{2}{*}{$\|$} & By method A & 1 & 1 & 1 & 0 & 0 & 0 & 2.33 \\
\hline & By method B & 1 & 0 & 0 & 0 & 0 & 1 & .5293 \\
\hline \multirow[t]{2}{*}{ III } & By method A & 2 & 2 & 0 & 0 & 0 & 0 & .6934 \\
\hline & By method B & 2 & 2 & 0 & 0 & 0 & 0 & .5632 \\
\hline \multirow[t]{2}{*}{ IV } & By method A & 2 & 2 & 1 & 0 & 0 & 1 & 5.33 \\
\hline & By method B & 1 & 1 & 0 & 0 & 0 & 2 & 2.65 \\
\hline \multirow[t]{2}{*}{ V } & By method A & 3 & 3 & 0 & 0 & 0 & 0 & 4.32 \\
\hline & By method B & 2 & 1 & 0 & 2 & 0 & 0 & 2.91 \\
\hline \multirow[t]{2}{*}{$\mathrm{Vl}$} & By method A & 3 & 3 & 2 & 1 & 0 & 0 & 4.85 \\
\hline & By method B & 1 & 2 & 1 & 0 & 0 & 0 & 2.91 \\
\hline
\end{tabular}

Stage $\mathrm{I}=2$ classic radars

Stage $\|=1$ classic radar, 1 staggered radar, 1 jittered radar

Stage III $=2$ classic radars, 2 staggered radars

Stage IV $=2$ classic radars, 2 staggered radars, 1 jittered radar

Stage $V=3$ classic radars, 3 staggered radars,

Stage $\mathrm{VI}=3$ classic radars, 3 staggered radars, 2 jittered radars

To evaluate the proposed algorithm, its efficiency must compare with the other multiparameter algorithms. In [5], a multi-parameter pulse separation algorithm compares with the TOA-based processing algorithm. So, to evaluate the quality performance of the proposed sequence search method, it has been compared with the one-parameter sequence search method based on the TOA for different scenarios according to Table 2 . In this table, in several steps for different scenarios, the proposed pulse separation method based on the parameters including RF, PW, and TOA (method A) is implemented and compared with the pulse separation method based on only-TOA processing (method B) in the sequence search. 
The proposed method has a better quality of data processing at mostly equal processing time by analyzing the table results. Besides, the proposed method has a better performance quality than the multi-parameter pulse separation method presented in [5] that both algorithms are comparing with the only TOA-based processing algorithm. And also, the proposed algorithms do not require additional processing like staggered sequence analysis.

In the next step, to compare the proposed method performance with the only-TOA processing algorithm performance, at first, these methods are implemented; then, the percentage of correct pulse separation is calculated in Fig. 7. The input data of both algorithms are the PDWs parameters of the received pulses. These pulses receive from all surroundings emitters, and the number of the emitters changes between 3 and 15 .

\section{Conclusions and future work}

In this paper, an improved method was implemented and investigated that separates the interleaved radar pulse trains. Also, the proposed method uses the time of arrival information simultaneously with the other parameters for the pulse deinterleaving operation. Using the developed sequence search in the proposed pulse separation method, the staggered and jittered sequences were also well separated. By investigating the obtained results from the proposed algorithm, it was possible to separate the emitters in the same direction (equal angle of arrival) with different pulse repetition interval characteristics. The proposed algorithm made it possible to use the TOA information efficiently in the radar pulses separation, which concluded in the simulation results. Also, investigation in the following aspects is suggested for future work, as follows.

- Study of methods for extracting identification parameters from a separated pulse sequence.

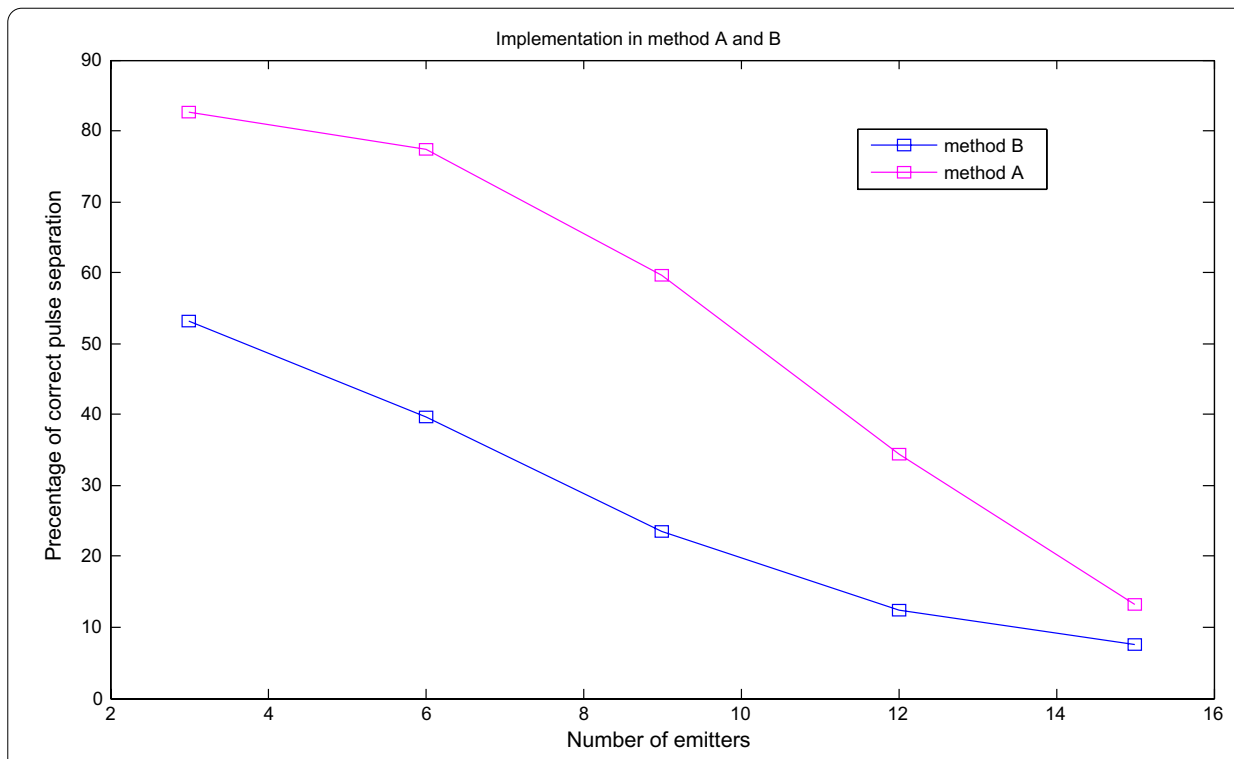

Fig. 7 Percentage of the correct pulse separation of method $A$ (the proposed pulse separation method based on RF, PW, and TOA) and method B (the pulse separation method based on only TOA) 
- Study of the emitter table updating by comparing the current and previously separated emitters in the emitter table.

- Study the implementation of electronic warfare and e-attack interaction operations using the features of the extracted pulse sequences.

- Study in tracking and monitoring the surrounding radar emitters using the separated pulse sequence and the extracted pulse repetition intervals.

- Study the receiver performance that measures the pulse parameters and improving the precision and accuracy of the measured parameters such as ESM and ELINT.

\section{Abbreviations}

PDW: Pulse descriptor word; ESM: Electronic support measures; TOA: Time of arrival; PRI: Pulse repetition interval; AOA: Angle of arrival; PW: Pulse width; RF: Carrier frequency; PA: Pulse amplitude; SDIF: Sequential difference histogram; CDIF: Cumulative difference histogram.

\section{Acknowledgements}

Not applicable.

\section{Authors' contributions}

$\mathrm{HH}$ participated in mathematical design of the proposed method and its computer implementation. MK coordinated industrial application and raw data preparation and helped out for study. $\mathrm{HH}$ and MK have completed the first draft of this paper. All authors have read and approved the final manuscript.

\section{Funding}

Not applicable.

Availability of data and materials

All the data and computer programs are available.

\section{Declarations}

Competing interests

The authors declare that they have no competing interests.

\section{Author details}

${ }^{1}$ School of Electrical and Computer Engineering, Shiraz University, Shiraz, Iran. ${ }^{2}$ Department of Computer Engineering, Persian Gulf University, Bushehr, Iran.

Received: 6 January 2021 Accepted: 13 April 2021

Published online: 20 April 2021

\section{References}

1. C.L. Davies, P. Hollands, Automatic processing for ESM, in IEE Proceedings, vol. 129, Pt. F, no. 3 (1982), pp. 164-171

2. R.G. Wiley, ELINT: The Analysis of Radar Signals, 1st edn. (Artech House Radar Library, 2006).

3. N.J. Whittall, Signal sorting in ESM systems. IEE Proc. Part F 132(4), 226-228 (1985)

4. H.K. Mardia, New techniques for the deinterleaving of repetitive sequences, in IEEE Proceedings, vol. 136, Pt. F, no. 4 (1989)

5. D.J. Milojevic, B.M. Popovic, Improved algorithm for the deinterleaving of radar pulses, in IEEE Proceedings, vol. 139, Pt. F, no. 1 (1992), pp. 98-104

6. M.E. Kocamis, S.B. Mihcioglu et al., A clustering approach for radar warning receivers, in IEE Conference Publisher, 23nd Signal Processing and Communications Applications Conference (SIU) (2015)

7. U.I. Ahmed et al., Comprehensive review of pulse repetitions interval (PRI) classification schemes, in IEEE Conference Publisher, Sixth International Conference on Aerospace Science and Engineering (ICASE) (2019)

8. U.I. Ahmed et al., Robust pulse repetition interval (PRI) classification scheme under complex multi emitter scenario, in IEEE Conference Publisher, 22nd International Microwave and Radar Conference (MIKON) (2018)

9. Y. Tang, M. He, X. Tang, J. Han, X. Fan, Method for automatically identifying PRI patterns of complex radar signals. IET J. 2019(20), 6975-6978 (2019)

10. C.L. Chowdhary et al., Analytical study of hybrid techniques for image encryption and decryption. Sensors 20(18), $5162(2020)$

11. C. Zhao et al., Radar signals sorting with Kohonen neural network, in IEEE Conference Publisher, 8th International Conference on Signal Processing (2006), pp 16-20 
12. J. Liu, J.P.Y. Lee et al., Online clustering algorithms for radar emitter classification. IEEE Trans. Pattern Anal. Mach. Intell. 27(8), 1185-1196 (2005)

13. E. Al-Hadhrami, M. Al-Mufti et al., Classification of ground moving radar targets using convolutional neural network, in IEEE article, 22nd International Microwave and Radar Conference (MIKON) (2018)

14. A.V. Kvasnov, Methodology of classification and recognition of the radar emission sources based on Bayesian programming. IET Radar Sonar Navig. 14(8), 1175-1182 (2020)

15. J. Tamang, J.D.D. Nkapkop, M.F. Ijaz, P.K. Prasad, N. Tsafack, A. Saha, Dynamical properties of ion-acoustic waves in space plasma and its application to image encryption. IEEE Access 9, 18762-18782 (2021)

16. K. Gençol, A two-stage deinterleaving technique for clustering of radar pulses, in IEE Conference Publisher, 25th Signal Processing and Communications Applications Conference (SIU), Antalya, Turkey (2017), pp. 1-574

17. M.K. Aslan, Emitter identification techniques in electronic warfare. M.S. thesis in Electrical and Electronics Engineering, Middle East Technical University, Ankara, Turkey (2006)

18. D.R. Wilkinson, A use of metric techniques in ESM data processing. IEE Proc. Commun. Radar Signal Process. 132 $121-125(1985)$

19. F. Fuhua, Y. Xuezhong, Improved method for deinterleaving radar pulse trains with stagger PRI from dense pulse series, in IEE Conference Publisher, 2nd International Conference on Signal Processing Systems (ICSPS) (2010)

20. Z. Ge, X. Sun, W. Ren, W. Chen, G. Xu, Improved algorithm of radar pulse repetition interval deinterleaving based on pulse correlation. IEEE Access 7, 30126-30134 (2019)

21. A. Erdogan et al., Pulse on pulse deinterleaving radar algorithm, in IEEE Conference Publisher, 10th Annual Computing and Communication Workshop and Conference (CCWC), Las Vegas (2020)

22. U.I. Ahmed et al., Comprehensive review of pulse repetitions interval (PRI) classification schemes, in IEEE Sixth International Conference on Aerospace Science and Engineering (ICASE) (2019)

23. M.F. Ijaz, M. Attique, Y. Son, Data-driven cervical cancer prediction model with outlier detection and over-sampling methods. Sensors 20(10), 2809 (2020)

24. M. Jawad, Y. Iqbal, N. Sarwar, PRI characteristics analysis under complex environment of spurious and missing observations, in IEEE 17th International Bhurban Conference on Applied Sciences and Technology (IBCAST) (2020)

25. J.A.V. Rogers, ESM processor system for high pulse density radar environments. IEE Proc. Commun. Radar Signal Process. 7, 621-625 (1985)

26. H.K. Mardia, Adaptive multidimensional clustering for ESM, in IEE Colloquium on signal processing for ESM systems, Digest:81988/62 (1988), pp. 5/1-5/4

27. O. Torun, M.B. Kocamış et al., Deinterleaving of radar signals with stagger PRI and dwell-switch PRI types, in IEEE Conference Publisher, Computer and science, 25th Signal Processing and Communications Applications Conference (SIU) (2017)

28. A.W. Ata'a, S.N. Abdullah, Deinterleaving of radar signals and PRF identification algorithms. IET Radar Sonar Navig. 1(5), 340-347 (2007)

29. F. Fan, X. Yin, Improved method for deinterleaving radar pulse trains with stagger PRI from dense pulse series, in 2nd International Conference on Signal Processing Systems; 5-7 July 2010; Dalian, China. New York, NY, USA. IEEE, pp. 250-253

30. S. Sridharan, N.N.S.S.R.K. Prasad, R. George, M. Brindha, Improved pulse repetition interval (PRI) deinterleaving for electronic support measure (ESM) receiver, vol. 2, no. 3, ISSN(PRINT):2394-3408, (ONLINE):2394-3416 (2015)

31. Y. Liu, Y. Chen, S. Sun, A radar signal sorting algorithm based on PRI, in IEEE Conference Publisher, 19th International Symposium on Communications and Information Technologies (ISCIT) (2019)

32. Y. Liu, Q. Zhang, Improved method for deinterleaving radar signals and estimating PRI values. IET Radar Sonar Navig. 12(5), 506-514 (2018)

33. Y. Xi, Y. Wu, X. Wu, K. Jiang, An improved SDIF algorithm for anti-radiation radar using dynamic sequence search, in IEEE Conference Publisher, 36th Chinese Control Conference (CCC) (2017)

34. C. Chen, Z. Liu et al., Traffic flow prediction based on deep learning in industrial internet of vehicles, IEEE Trans. Intell. Transp. Syst. (2020). https://doi.org/10.1109/TITS.2020.3025856

35. A. Zhou, S. Wang et al., LMM: latency-aware micro-service mashup in mobile edge computing environment. Neural Comput. Appl. 32, 15411-15425 (2020)

36. X. Xu, X. Liu et al., Joint optimization of resource utilization and load balance with privacy preservation for edge services in $5 \mathrm{G}$ networks. Mob. Netw. Appl. 25, 713-724 (2020)

37. J. Liu, W. Wang et al., Role of gifts in decision making: an endowment effect incentive mechanism for offloading in the loV. IEEE Internet Things J. 6(4), 6933-6951 (2019)

38. D.R. Wilkinson, A.W. Watson, Use of metric techniques in ESM data processing. IEE Proc. Radar Radionavig. 132, 229 (1985)

\section{Publisher's Note}

Springer Nature remains neutral with regard to jurisdictional claims in published maps and institutional affiliations. 